evolutionary theory to all aspects of research on this subject. In the first half, Rose deals directly with evolutionary issues, from explaining the theory, through to describing experimental tests of the evolutionary theories and genetic mechanisms for the evolution of altered life span. Particularly interesting are the experiments of Rose and others with Drosophila melanogaster, where selection for late reproductive ability results in increased life span. The finding is in accordance with the 'antagonistic pleiotropy' theory, which comes principally from a landmark paper by George Williams that was published in 1957. The idea is that genes conferring a fitnessadvantage early in life will be favoured even if they cause the deleterious effects of senescence later on. The second half of the book deals briefly with the comparative biology of ageing, organismal theories of ageing, and cellular and molecular theories. Some of this material is a bit dated.

Biology of Aging is aimed at those requiring an introduction to the field. The style is engaging and light, and the book covers the main subject areas fairly well. Arking leads off with a comparative approach, dwells in some detail on human senescence, dips into various theories on the mechanisms of ageing, and concludes with a synthetic view of

\section{Eccentric behaviour?}

\section{David W. Hughes}

Comet Halley: Investigations, Results, Interpretations. Vol. 1: Organization, Plasma, Gas. Vol. 2: Dust, Nucleus, Evolution. Edited by J. W. Mason. Ellis Horwood: 1990. Pp.295 (Vol. 1), 275 (Vol. 2). Each $£ 62, \$ 105.50$.

AFTER the 1910 apparition of Halley's comet, astronomers had to wait some twenty-one years before Nicholas $T$. Bobrovnikoff at the Lick Observatory produced a comprehensive review of the results. Things have speeded up and expanded since then, and the thirtieth recorded apparition of the comet in 1985-1986 saw more of the world's scientists turn their attention to this once-ina-lifetime visitor than ever before. We must congratulate John Mason on getting into print within four years.

Mason has assembled 39 detailed review articles into two volumes by calling on the expertise of a host of the world's top cometary experts and space scientists. The two volumes are targeted at the professional cometary astronomer. Other astronomers, including amateurs, will still have to wait for a more digesti- ageing as a "genetically determined, environmentally modulated, event-driven process". What this means, in simple English, is that Arking sensibly recognizes that the control of longevity is best understood as a blend between genetic factors, such as genes that programme the efficacy of cell maintenance systems like DNA repair, and stochastic or environmental factors, such as those causing the lesions needing to be repaired. On the negative side, the book draws rather heavily on a few key sources, is weak on life-history theory, and spells people's names wrong rather often. But the pluses well outweigh the minuses, and the book should encourage bright students to study ageing further.

One swallow does not make a spring. Aristotle wrote that, too, in his Nichomachean Ethics. Similarly, three good new books on ageing do not herald the end of gerontology's problems. They are, however, a healthy sign. Understanding the mechanisms of ageing is one of the greatest challenges confronting biological and medical research. A great deal of hard science and a good measure of interdisciplinary cooperation will be needed to crack it.

Thomas B. L. Kirkwood is at the National Institute for Medical Research, The Ridgeway, Mill Hill, London NW7 1AA, UK.

ble account of the changes in our understanding of comets brought about by our observations of Halley.

Between January and March 1986, Halley's comet passed inside the Earth's orbit. For over two years around this period, many of the world's great telescopes were turned towards the comet. The astronomers in 1910 were confined to the use of the narrow visual band of the electromagnetic spectrum; 1986 saw full use of the ultraviolet and the infrared parts. Advantage was also taken of the new technologies of the space-age, an armada of six spacecraft rushing past the comet as it moved through the descending node of its orbit. No longer were cometary observers compelled to flatten their quarry onto the plane of the sky. They could now, for a brief moment, add the third dimension. They could also escape the confines of Earth and move very much closer.

The main success of the enterprise was to achieve the first-ever imaging of the cometary nucleus. The model of the central, kilometric sized, dirty snowball as the fount of all cometary activity, placed on a firm foundation by Fred L. Whipple 35 years before, was transformed into a reality.

The new collection of reviews is divided into six sections. The first looks at the organization and coordination of the scientific effort, involving the coopera- tion of the European, Soviet and Japanese space agencies and the setting up of the International Halley Watch. The second section concentrates on plasmas and the complicated interaction between the solar-wind plasma streaming away from the Sun and the cometary plasma escaping from the vicinity of the sublimating snowy nucleus. Many fine images of disconnection events in the plasma tail are shown and much is made of the influence of the cometary plasma on the interplanetary magnetic field. The third section deals with the gas emitted by the comet, its flux, composition and spatial distribution. Next comes a section on the dust, with chapters on tails, jet morphology, size distribution, meteoroid streams and composition. The fifth section considers the nucleus, its shape, roughness, activity, mass, surface temperature distribution and spin state. The final section investigates the orbital evolution of comet Halley over the last few millennia and its physical evolution during that time.

We are presented with a detailed overview of what was discovered about the comet during its last apparition. The two volumes are superbly produced, lavishly illustrated, well referenced and usefully indexed. But to my mind there are two important deficiencies. First, too little attention is drawn to our ignorance. For example, the first tantalizing glimpses of a cometary nucleus resulted in a resolution of only about 150 metres and we have no idea as to the macro-properties of the dust and snow on the active and inactive regions of the comet's surface. Also, the spacecraft, moving at 150,000 miles per hour, could quantify only the fragments of the emitted cometary molecules and dust particles, and our detailed knowledge of their composition is negligible. The mass of the nucleus is known to a factor of four at best, and its internal dust, snow and density structure is still a complete mystery.

Second, there is no mention of the relationship between Halley's comet and all the other comets. We have benefited greatly from a fleeting glimpse of Halley, but have no firm idea about how typical it is. We are forced to use it in the calibration and interpretation of other cometary events, but it might be in itself quite unusual: we just do not know.

David $W$. Hughes is in the Department of Physics, University of Sheffield, Sheffield S3 $7 R H$, UK.

\section{Correction}

Hormones edited by Etienne-Emile Beaulie and Paul A. Kelly (reviewed in Nature 351 , 362; 30 May 1991) is distributed by Chap. man and Hall in the United Kingdom, the Commonwealth, the United States and Cana$\mathrm{da}(£ 47.50, \$ 62.50$ (pbk)). Hermann has all other European rights. 